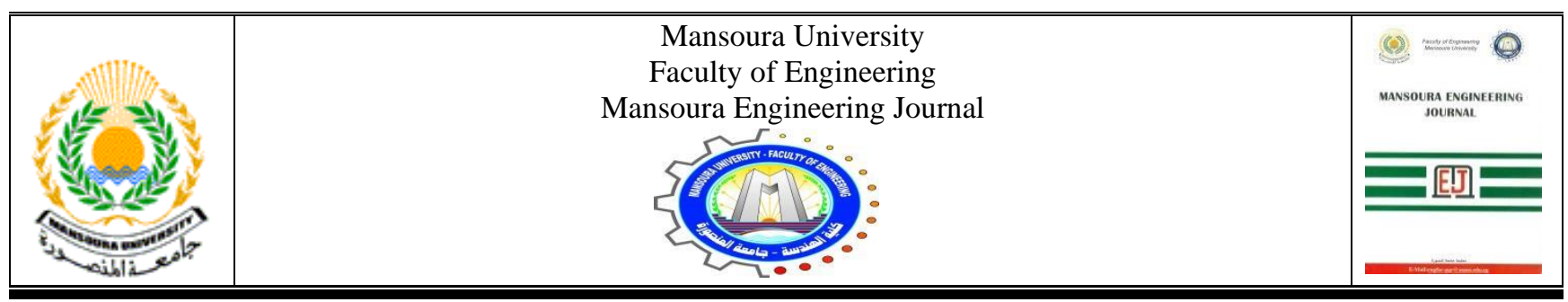

\title{
Automated Deep System for Joint Liver and Tumor Segmentation Using Majority Voting
}

\author{
A. Elnakib*, N. Elmenabawy and H.S. Moustafa
}

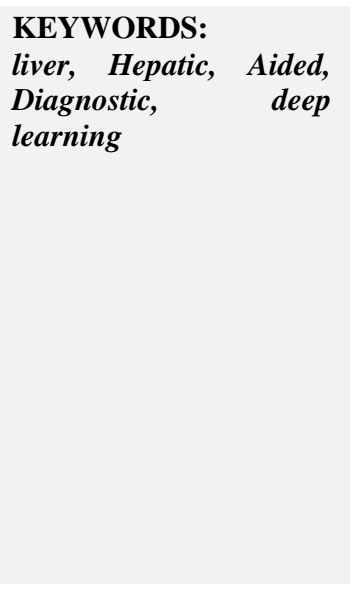

\begin{abstract}
In this paper, a system based on deep learning and majority voting is proposed for joint segmentation of the liver and hepatic tumors. The proposed system is composed of three steps. First, deep learning is utilized to extract deep features that describe the Computed Tomography (CT) images as well as cancerous nodules, using three different Convolutional Neural Networks (CNNs), i.e., VGG16-Segnet, Encoder-Decoder (ED)-Alexnet, and Resnet18. Second, a classification step using the extracted deep learning features is performed for each investigated network. To produce the final liver and hepatic tumor segmentation, the last step applies a majority voting technique to fuse the three utilized CNN outputs. To test the performance of the proposed system, the MICCAI LITS challenge database is used, composed of $130 \mathrm{CT}$ volumes with a total of 16,917 cross-section images. The proposed system achieves Dice Similarity Coefficients (DSCs) of $94 \%$ and $76 \%$ for liver and lesion segmentations, respectively. Comparison with the related methods confirms the promise of the proposed system for joint liver and tumor segmentations.
\end{abstract}

\section{INTRODUCTION}

$\mathrm{L}$ IVER cancer is the second leading cause of global cancer mortality (after lung cancer). In Egypt, the liver cancer is more epidemic. According to the National Cancer Registry Program (NCRP) in Egypt, around 167 per 100,000 had diagnosed with liver cancer, representing the leading cancer incidence rate of $23.8 \%$ [1]. Clinically, the early diagnosis and assessment of tumor volumes have shown an ability to reduce the mortality rate. However, the visual assessment of Computed Tomography (CT) images is normally time-consuming and suffers from inter- and intra-observer variability. To solve these problems, different Computer Aided

Received: (31 August, 2020) - Revised: (11 November, 2020) - Accepted: (27 November, 2020)

* Corresponding author: A. Elnakib, is with the Electronics and Communications Engineering Department, Faculty of Engineering, Mansoura University, 35516 Mansoura City, Egypt (e-mail: nakib@mans.edu.eg)
Diagnostic (CAD) systems have been developed to detect, segment, and diagnose the liver cancer.

Developing efficient CAD systems for liver segmentation, a primary step for more focused clinical analysis [2], and for hepatic tumor segmentation is a very challenging problem since that the CT liver images usually have low soft-tissue contrast and suffer from noise and other artifacts. In addition, tumor segmentation is more challenging due to the significant variations in the size of lesions, their shapes, intensities, textures, and how many times they occurred across different patients. Throughout literature, different methodologies have been utilized for liver segmentation and/or for liver cancer segmentation. These methods can be categorized as traditional methods or deep learning methods. Traditional methods usually extract features, e.g., intensity, texture, shape, from liver CT

N. Elmenabawy, is with the Electronics and Communications Engineering Department, Faculty of Engineering, Mansoura University, 35516 Mansoura City,Egypt (e-maileng_nermeena@yahoo.com).

H. S. Moustafa, is with the Electronics and Communications Engineering Department, Faculty of Engineering, Mansoura University, 35516 Mansoura City, Egypt (e-mail: hossam_moustafa@hotmail.com). 
images and use a classifier based on these features to perform the segmentation process. On the other hand, deep learning methods usually use Convolutional Neural Networks (CNN) to perform the segmentation. A CNN model contains a set of convolutional layers to encode the low- and high-level features of the liver images. Throughout the literature, CNN models show a promising result for liver and tumor segmentation. However, current deep learning methods suffers from the following limitations:

- They require a large database for efficient training

- Most of them are computationally expensive, especially on the training phase

- There is a need to develop new systems/ideas to improve the segmentation accuracy

To overcome these limitations, a deep learning CAD system for simultaneous liver and liver cancer segmentation is proposed. The proposed system adopted transfer learning, where the weights of the convolutional encoder layers are transferred without training, to eliminate the need for huge training data and significantly reduce the computations. The main contributions/features of this work are as follows:

- Adopting transfer deep learning of different CNN architectures for simultaneous liver and tumor segmentation

- Applying joint liver and lesion segmentation using parallel CNN models

- Smart fusion of three CNN architectures to improve the overall performance

- Performance evaluation using the challenging MICCAI'2017 LITS database

In additional to the accurate automated tumor segmentation, the proposed system shows an ability to accurately segment the liver, evidenced by the highest achieved Dice Similarity Coefficient (DSC) metric, compared to the related work for tumor segmentation. The rest of this paper is organized as follows. Section II overviews the related liver and tumor segmentation methods. Section III illustrates the proposed system for simultaneous liver and tumor segmentation. Section IV details the experimentations, the comparative results, and the related discussions. Finally, section V concludes the paper.

\section{LITERATURE REVIEW}

This section overviews the related traditional and deep learning techniques for liver and tumor segmentation.

\section{A. Traditional approaches}

Traditional techniques for liver and tumor segmentation perform feature extraction, followed by classification. For example, Bastian et al. [3] used intensity feature and applied a super-pixel Simple Linear Iterative Clustering (SLIC) approach and an AdaBoost algorithm to segment the liver, achieving a Dice rate of $92.13 \%$ on 16 abdomen CT test images. Ali et al. [4] used the first order statistical features of the liver image to extract the CT liver boundary. Then, a k-Mean classifier based on the distance and color is used for lesions classification. Chang et al. [5] segmented the tumors using a region growing algorithm. A binary logistic regression analysis, based on the extraction of texture, shape, and kinetic curve features, is performed to classify the segmented tumors into benign or malignant. The main limitation of traditional approaches is that the extracted features may not be sufficient to describe the complexity of the tumor segmentation problem. Therefore, more sophisticated features should be investigated.

\section{B. Deep learning approaches}

Unlike traditional approaches, deep learning makes full use of the database, i.e., the $\mathrm{CT}$ images and the corresponding Ground Truth (GT) segmentations by the radiologists, to train a set of convolutional features/parameters that can describe the segmentation problem. Usually, deep approaches use a huge number of parameters (i.e., millions) to learn the problem. In the last decade, deep Convolutional Neural Networks (CNN) architectures have achieved outstanding performance in liver and lesions segmentations. This section overviews the related deep liver and lesion segmentation approaches.

\section{1) Separate liver or lesion segmentation approaches}

Liver segmentation is a preliminary step in many CAD systems for liver cancer or other liver diseases. For example, Hu et al. [6] segmented the liver, based on a 3D CNN model, to provide an initial prior segmentation. The surface of the prior segmentation was adaptability evolved based on image appearance to provide the final segmentation. On the other hand, different deep methods for lesion segmentation, a more challenging task, were implemented throughout the literature. For example, Bi et.al [7] used a deep residual networks (Resnet) [8] for liver lesions segmentation. Han et.al [9] built a CNN model to segment the liver lesion automatically. Since this work deal with joint liver and lesion segmentation, a more focused overview of the related joint methods is provided below.

\section{2) Joint approaches for liver and lesion segmentations}

Regarding joint liver and lesions segmentations, these methods can be categorized as one stage or cascaded. Below, we will briefly overview each category.

One stage deep learning approach attempt to segment the liver and its lesions using one deep CNN stage. For example, Korabelnikov et.al [10] used a segmentation framework which is consisting of a pre-processing step, a pixel-wise classification using pre-trained CNN (Alexnet) and smoothing and thresholding as post-processing of the obtained binary segmented image. Badrinarayanan et al. [11] used the VGGSegnet model directly for joint liver and tumor segmentation.

Cascaded deep learning approaches attempt to segment the liver and lesion in more than one stage, arranged in a cascaded manner. For example, Bellver et.al [12] used two cascaded VGG16 [13] models; the first one produces the liver segmentation, to be the input of the second VGG16 model, which produces the lesion segmentations. Vorontsov et.al [14] used a model for the joint segmentation of the liver and lesions from CT liver images. The model was built from two cascaded fully convolutional networks (Unet-like) [15] that were trained together end-to-end to segment the liver and lesions, respectively. In summary, different traditional and deep learning methods have been used either for liver segmentation 
or tumor segmentation or both. However, there is a need to investigate better methods to improve the accuracy. To achieve this task, the proposed framework fuses three different CNN networks, adopting a transfer learning model using a majority voting scheme.

\section{METHODS}

The raw liver CT images are input to the proposed framework without any preprocessing steps. The proposed framework, see Fig. 1, processes a raw image through three stages. First, features are extracted by investigating three different CNN architectures. Second, pixel-wise classification layer is applied. Finally, a majority voting scheme is applied. The details of each stage is illustrated below (Fig.1).

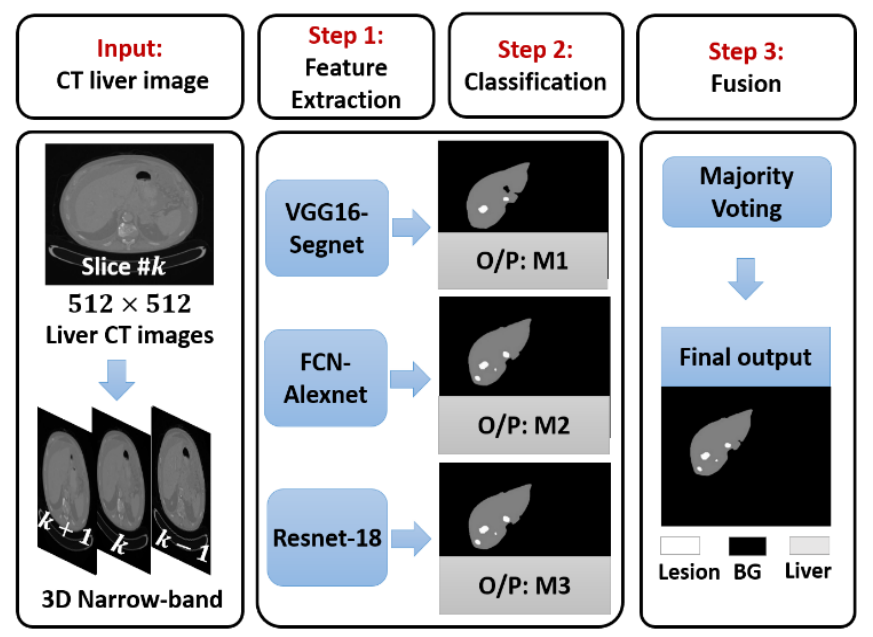

Fig. 1. Proposed framework for liver and lesion segmentation. The 3D narrow-band image information is input to three deep feature extraction CNN models, where their classification outputs are fused.

\section{A. Database Collection}

We used the LiTS dataset for the training and testing of the proposed system. The LiTS datasets consist of 130 contrastenhanced abdominal CT scans from several different clinical sites with different scanners and protocols. The dataset has largely varying spatial resolution and fields-of-view. The axial slices of all scans have an identical size of $512 \times 512$, but the number of slices in each scan differs greatly and varies between 42 and 1026 resulting in a total of 16,917 CT cross-sections. The in-plane resolution ranges from $0.60 \mathrm{~mm}$ to $0.98 \mathrm{~mm}$, and the slice spacing from $0.45 \mathrm{~mm}$ to $5.0 \mathrm{~mm}$. Some images contain imaging artifacts (e.g. metal artefacts), which are present in real life clinical. The size of the tumors vary between $38 \mathrm{~mm}^{3}$ and $349 \mathrm{~cm}^{3}$ [16]. The average tumor-liver intensity difference is defined as the average absolute difference between the liver voxel Hounsfield Unit (HU) values and the tumor voxel values. They vary between 0 and 98 and have a mean of $31.94(\mathrm{SD}=20)$ and a median of 29.61 [16], [17].

\section{B. $3 D$ narrow band}

To account for the 3D anatomy of the tumor, the 3D narrow band information of each slice is taking into account. As shown in Fig. 1, for the current processed slice of the index $k$, the adjacent neighbors of indices $k-1$ and $k+1$ are input to the feature extraction block (i.e., the $\mathrm{CNN}$ model).

\section{Feature extraction}

Pre-trained CNN models are adopted for feature extraction. More specifically, VGG16-SegNet, Resnet-18, and EDAlexnet to extract liver and lesions features. The details of each network are illustrated below.

VGG16-Segnet model is composed of the VGG16 encoder network, a corresponding decoder network, and a final pixelwise classification layer [11]. In this work, we used the pretrained VGG16 encoder network model, which is composed from only the convolutional layers with no fully connected layers (i.e., 13 convolutional layers in Fig. 2) that were pretrained on 1.2 million images of the ImageNet ILSVRC2014 dataset [13] (see Table 1). Each encoder layer has a corresponding decoder layer and hence the decoder network is composed of 13 layers. The final decoder output is fed to a softmax classifier to produce class probabilities for each pixel independently. More details about the VGG16-Segnet model can be found in [11].

ED-Alexnet model is composed of the Alexnet encoder network, a corresponding decoder network, and a final pixelwise classification layer [18]. The Alexnet encoder is composed of five convolutional layers (see Fig. 3) that were trained on 10 million images of the ImageNet ILSVRC-2012 dataset (see Table I). The decoder is composed of one deconvolutional layer that corresponds to the deconvolution of the encoder five layers. The final decoder output is fed to a soft-max classifier to produce class probabilities for each pixel independently. More details about the ED-Alexnet model can be found in [18].

Resnet18 model is composed of an 18 weighted layers encoder, a decoder, and a pixel classification layer [8]. This model is pretrained on 1.2 million labeled high-resolution images belonging to 200 categories. Table 1 summarizes the details of the pre-trained VGG16, Alexnet and Resnet-18 models, their training data, and their structures.

\section{Classification}

A pixel-wise classifier is used after each model's decoder to label the segmented output image. The pixel-wise classifier performs softmax classification. Three softmax nodes are utilized per each image pixel, providing the probabilities of the three labels: lesion, liver, or background [19], as follows:

$$
\sigma\left(x_{i}\right)=\frac{e^{x_{i}}}{\sum e^{x_{i}}}
$$

where $x_{i}$ denotes the input to the softmax node $i$ and $\sigma($. denotes the output probability of the softmax node. The final output label for each pixel (i.e., lesion, liver, or background) corresponds to the softmax node of the largest probability.

\section{E. Majority voting scheme}

The majority voting scheme [20] is applied to the binary classified outputs of the three utilized CNN models (VGG16Segnet, ED-Alexnet and Resent-18). The details of the majority voting scheme are illustrated Fig. 4. Let $A C C_{\text {net }}^{\text {label }}$ denote the network's accuracies for each label, where Label $\in\{B G$, Liver, Tumor $\}$ and net $\in$ \{VGG16-Segnet, ED-Alexnet, Resnet-18\}. 


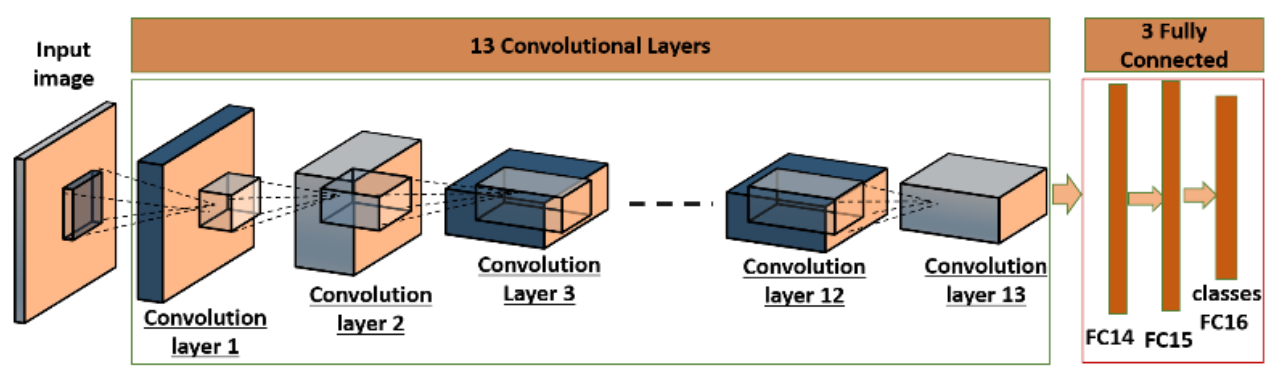

Fig. 2. The VGG16 network architecture.

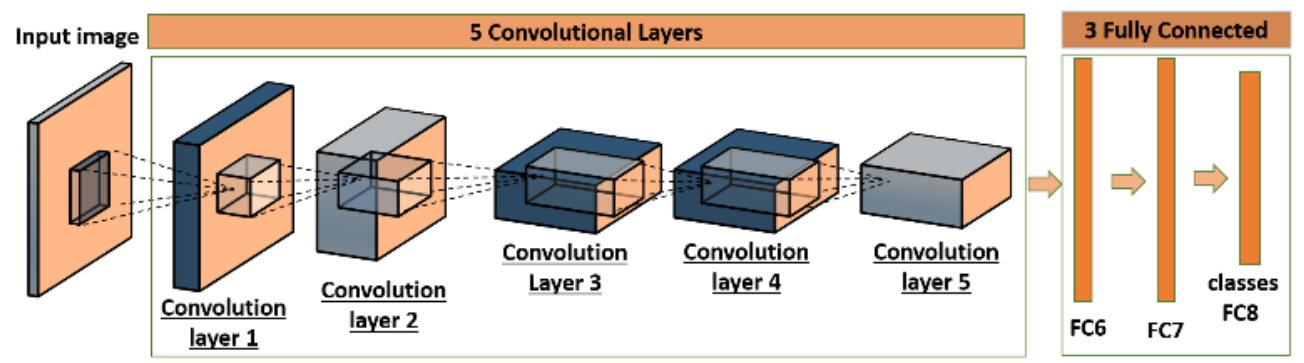

Fig. 3. The Alexnet network architecture

TABLE 1

SUMMARY OF THE PRE-TRAINED VGG16, RESNET-18 AND ALEXNET CNN MODELS. THE SYMBOL “\#” IN THE TABLE INDICATES THE NUMBER, FC DENOTES FULLY CONNECTED, AND CL DENOTES CONVOLUTIONAL LAYERS

\begin{tabular}{c||c||c||c|c||c||c||c}
\multirow{1}{*}{\multicolumn{1}{c||}{$\begin{array}{c}\text { CNN } \\
\text { model }\end{array}$}} & Database & \multicolumn{2}{c||}{ CNN Model Description } & \multicolumn{2}{c}{ Pre-trained Network Parameters } \\
\cline { 2 - 6 } & Input Size & \# CL & $\begin{array}{c}\text { \#FC } \\
\text { Layers }\end{array}$ & $\begin{array}{c}\text { \#Images } \\
\text { per million }\end{array}$ & \#Subjects & $\begin{array}{c}\text { Parameter size } \\
\text { per million }\end{array}$ \\
\hline VGG-16 & $\begin{array}{c}\text { Imagenet } \\
\text { (ILSVRC2014) }\end{array}$ & $224 \times 244 \times 3$ & 13 & 3 & 1.2 & 200 & 138.0 \\
\hline Resnet-18 & $\begin{array}{c}\text { Imagenet } \\
\text { (ILSVRC2014) }\end{array}$ & $224 \times 244 \times 3$ & 17 & 1 & $1 . .2$ & 200 & 138.0 \\
\hline FCN-Alexnet & $\begin{array}{c}\text { Imagenet } \\
\text { (ILSVRC2012) }\end{array}$ & $244 \times 244 \times 3$ & 5 & 3 & 10.0 & +1000 & 62.4
\end{tabular}

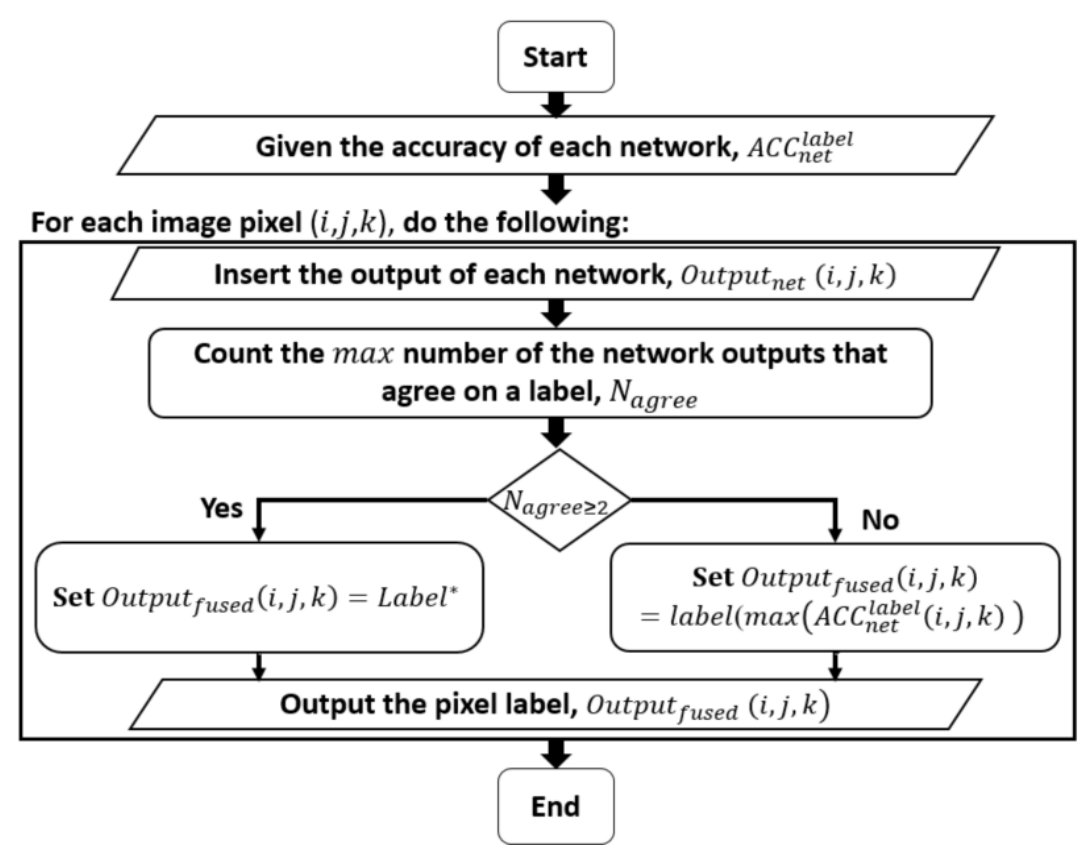

Fig.4. The majority vote scheme 
Let the coordinates of each image pixel be $(i, j, k)$, where $i$ the row index, $i \in\{1, \ldots, R\}, j$ is the column index, $j \in$ $\{1, \ldots, C\}, k$ is the image index, $k \in\{1, \ldots, K\}, R$ is the number of rows, $C$ is the number of columns, and $K$ is the number of the input images. Let Output $t_{n e t}(i, j, k)$ be the network output label at $(i, j, k)$, where Output $_{\text {net }}(i, j, k) \in\{\mathrm{BG}$, Liver, Tumor . Let Output $_{\text {fused }}(i, j, k)$ be the fused output label at $(i, j, k)$, where Output $_{\text {fused }}(i, j, k) \in\{\mathrm{BG}$, Liver, Tumor $\}$. As shown in Fig. 4, if the number of networks that agrees on a label, classified as abel $^{*}$, is more or equal to two, i.e., $N_{\text {agree }}>=2$, then the fused output label is assigned to this label, i.e., Output $_{\text {fused }}(i, j, k)=$ Label $^{*}$. Otherwise, a tie has reached, and the Output fused $(i, j, k)$ is selected as the label of the network of the highest accuracy, i.e., of the maximum

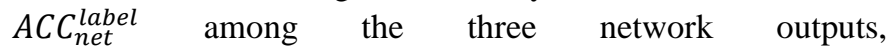
i.e., Output fused $(i, j, k)=\operatorname{label}\left(\max \left(\operatorname{ACC}_{\text {net }}^{\text {label }}(i, j, k)\right)\right.$.

Algorithm 1 summarizes the overall joint liver and lesion segmentation algorithm using majority vote technique.

Algorithm 1 Joint liver and lesion segmentation algorithm

Input: Input image of index $k$

Output: Output fused $_{\text {Image }}$

Step1: Formulate the 3D narrow band image, $I M_{3 D}$ by adding the two adjacent image neighbors at $k-1$ and $k+$ 1.

Step2: Extract features from $I M_{3 D}$ using the three pretrained networks, i.e., VGG16-Segnet, ED-Alexnet, and Resnet-18.

Step 3: Apply a pixel-wise classifier after each model's decoder

Step 4: Fuse the binary classifiers' outputs using the majority voting scheme in Fig.4, to produce the final fused output image Output fused $_{\text {. }}$

\section{F. Performance Metrics}

To test the performance of the proposed system, the Dice similarity coefficient (DSC) [21] is used, defined as two times the area of the intersection of A and B, divided by the sum of the areas of $\mathrm{A}$ and $\mathrm{B}$ :

$\operatorname{DSC}(\mathrm{A}, \mathrm{B})=(2 \times|\mathrm{A} \cap \mathrm{B}|) /(|\mathrm{A}|+|\mathrm{B}|) \times 100 \%(2)$

where $\mathrm{A}$ is the binary output labeled image, $\mathrm{B}$ is the binary ground truth image, and $|$.$| denotes the area. DSC can be also$ defined as follows:

$\mathrm{DSC}=2|\mathrm{~A} \cap \mathrm{B}| /(|\mathrm{A}|+|\mathrm{B}|)=2 \mathrm{TP} /(2 \mathrm{TP}+\mathrm{FP}+\mathrm{FN})$

where $\mathrm{TP}=$ True Positive, FP=False Positive, FN=False Negative. The better the segmentation is, the closer the DSC to 1 . The worst the segmentation, the closer the DSC to 0. Note that for liver segmentation, the label ' 1 ' denotes the object (liver) and the label ' 0 ' denotes the background, whereas for tumor segmentation, the label ' 1 ' denotes the object (tumor) and the label ' 0 ' denotes the background.

\section{EXPERIMENTAL RESULTS AND DISCUSSIONS}

In this section, the data specifications, the experimental setup, and the details and discussions of the experiments are illustrated.

\section{A. Experimental setup}

Deep learning training of the three utilized models (i.e., VGG16-Segnet, ED-Alexnet, and Resnet-18) is performed by initializing the layers in the down-sampling (encoder) with the pertained VGG16, Alexnet and Resnet parameters, whereas the parameters of the remaining layers of the three models are set to small random numbers with zero mean. The whole three networks are then fine-tuned using the LITS dataset in a pixelto-label way. The stochastic gradient descent (SGD) is utilized for training with a fixed learning rate of 10-3 and momentum of 0.9 . Before each epoch, the training set is shuffled and each mini batch of size of 200 images is then picked in order thus ensuring that each image is used only once in an epoch. The crossentropy loss as the objective function for training all the networks.

System evaluation is performed using the standard 5-fold cross-validation. For each fold, the 130 separate LITS scans are divided into 26 test subjects (20\% of the scans) and 104 training subjects ( $80 \%$ of the scans).

\section{B. Experimental results:}

In order to show the advantage of fusing the CNN models using the proposed majority voting scheme (proposed system), Fig. 5 provides a sample of the visual comparison results between the proposed system and using only one of the investigated CNN models (i.e., VGG16-Segnet, ED-Alexnet and Resnet-18). As shown in Fig. 6, ED-Alexnet provides the best performance between the individual investigated models for the segmentation of the liver as well as the tumor. However, the proposed fusing scheme provide a better visual match, to the Ground Truth (GT), than the best individual segmentation model (ED-Alexnet alone). To quantify these results, Table 2 details the comparison results in terms of the average DSC values over the whole test images. Consistent with the visual results in Fig 5, the proposed fusion scheme shows the ability to improve the performance over the best individual model (ED-Alexnet). It is remarkable here to note that although that the Alexnet model contains the least number of encoder layers (only 5 convolutional layers), it provides better performance that the competing VGG16-Segnet (13 layers) and Resnet-18 (18 layers) models. These may be due to the fine-tuning of the parameters of a smaller network is more efficient than that of larger networks, to avoid the overfitting problem. Note that the processing time of a test image to be segmented using our proposed system is 78 seconds.

In addition to the comparison to the individual networks, we also compared the proposed system with a weighted softmax-accuracy fusion of the three networks. This is done by multiplying the softmax value with the network accuracy then take the maximum as a winner. As shown in Table 2, the proposed majority voting achieves better results than the 
weighted softmax-accuracy fusion. This highlight the advantage of the proposed fusion scheme.

To investigate the advantage of the proposed system, its results are further compared to the related methods on the same LITS competition database effectiveness (see Table 2). The proposed fusion scheme achieves the highest performance for tumor segmentation among all the compared methods. However, the liver segmentation results are still slightly below the compared methods. The superior performance for tumor segmentation may be due to using the proposed utilized 3D narrow band information as an input to the CNN models. The 3D narrow band information takes into account the 3D extension of the tumor shape on both neighbor slices of the test image. For the liver segmentation, since the liver is of relatively large size than the tumor, this advantage will be less effective. In the future, we will investigate how to further improve the liver segmentation. However, in the specific case of liver cancer, the accurate tumor segmentation is the most clinically important step to stage the tumor and provide the proper medication.

\section{CONCLUSION}

In this paper, we proposed a framework for joint liver and lesions segmentation using the pre-trained CNN models (VGG16-Segnet, ED-Alexnet, and Resnet-18). The network outputs are fused using a majority vote scheme that efficiently improves the performance over each individual network. In additional, the proposed system shows superior performance for liver tumor segmentation, over the related compared methods. These results show the promise of the proposed system for joint liver and tumor segmentation. In the future, more data will be investigated to check the system robustness and more CNN models will be tested to improve the performance.
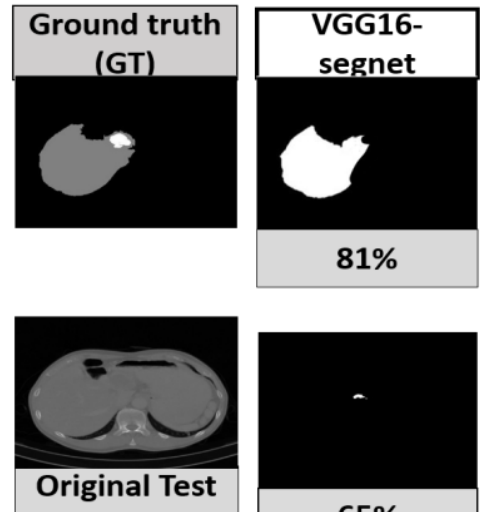

Image
$81 \%$

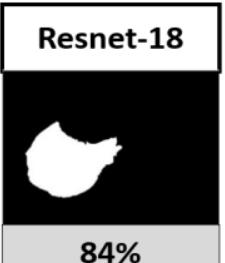

$84 \%$

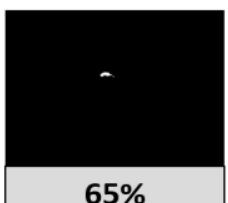

$65 \%$

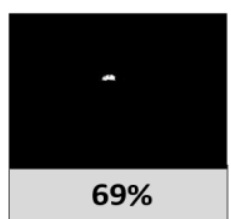

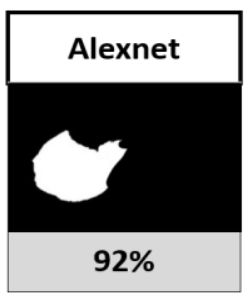
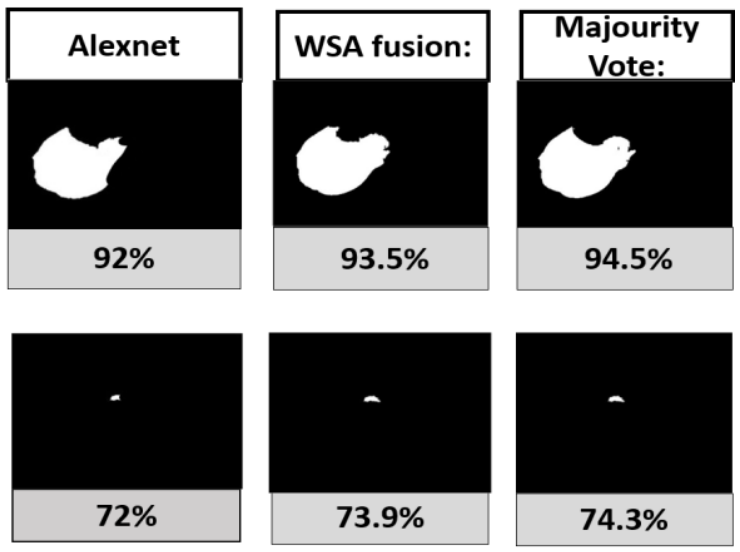

Fig. 5. Visual comparison segmentation results for a sample test CT image. Column I represents the output results for liver and tumor segmentation using VGG16-segnet, Column 2 represents the output results for liver and tumor segmentation using Resnet-18, Column3 represents the output results for liver and tumor segmentation using Alexnet, Column 4 represent the output results for liver and tumor segmentation using weighted softmax accuracy fusion

Last column represents the output results for liver and tumor segmentation using Majority vote, Numbers represents the achieved dice

TABLE 2.

A COMPARISON RESULTS FOR LIVER AND TUMOR SEGMENTATION BETWEEN EACH UTILIZED DEEP LEARNING MODEL, THE PROPOSED MAJORITY VOTING SYSTEM, AND OTHER RELATED METHODS

\begin{tabular}{l||l||l}
\multirow{2}{*}{\multicolumn{1}{l||}{ CNN Model }} & Liver & Tumor \\
\cline { 2 - 3 } & DSC & $50.1 \%$ \\
\hline Cascaded ResNet [7] & $95.1 \%$ & $43.0 \%$ \\
\hline Base network is VGG16 [12] & $96.0 \%$ & $52.0 \%$ \\
\hline Cascaded Unet-like [14] & $95.0 \%$ & $62.4 \%$ \\
\hline CDNN-Alexnet with preproceeing [22] & $90.4 \%$ & $69.7 \%$ \\
\hline Segnet-VGG16 & $82.3 \%$ & $71.4 \%$ \\
\hline Resnet18 & $85.2 \%$ & $74.7 \%$ \\
\hline ED-Alexnet & $93.5 \%$ & $75.2 \%$ \\
\hline Weighted softmax accuracy Fusion & $93.7 \%$ & $76.5 \%$ \\
\hline Proposed Majority Voting System & $94.2 \%$ &
\end{tabular}

\section{REFERENCES}

[1] Amal.S Ibrahim, Hussein.M Khaled,Nabiel.NH ,et.al."Cancer incidence in Egypt: results of the national population-based cancer registry program," Journal of cancer epidemiology vol. (2014),2014.

[2] L.Huang ., M Weng. ., H.uai.., et.al. "Automatic liver segmentation from CT images using single-block linear detection". BioMed research international, vol. (2016),2016.
[3] M. Barstugan. R. Ceylan., M. Sivri, et.al.2018“Automatic liver segmentation in abdomen CT images using SLIC and AdaBoost algorithms". In Proceedings of the 2018 8th International Conference on Bioscience, Biochemistry and Bioinformatics, pp. 129-13. ACM.

[4] A.H. Ali.and E.M. Hadi." Diagnosis of Liver Tumor from CT Images Using First Order Statistical," International Journal of Engineering Trends and Technology (IJETT) - Vol. (20), 2015. 
[5] C.C.Chang, , H.H, Chen, Y.C. Chang, et.al." Computer-aided diagnosis of liver tumors on computed tomography images". Computer methods and programs in biomedicine, vol. (145), pp. 45-51,2017.

[6] P. Hu, F.Wu, J. Peng, et.al. "Automatic 3D liver segmentation based on deep learning and globally optimized surface evolution, "Physics in Medicine \& Biology, vol.61(24), pp.8676,2016.

[7] L. Bi, J. Kim, J, A. Kumar, et.al. "Automatic liver lesion detection using cascaded deep residual networks. arXiv preprint arXiv:1704.0270,2017.

[8] K. He, X. Zhang, S. Ren and J. Sun,2016 "Identity mappings in deep residual networks," in European Conference on Computer Vision. Cham, Springer, vol. (9908), pp. 630-645

[9] X. Han, "Automatic Liver Lesion Segmentation Using a Deep Convolutional Neural Network Method" arXiv:1702.05747, 2017.

[10] A.N. Korabelnikov, A.V. Kolsanov, S.S. Chaplygin, P.M. Zelter2, K.V. Bychenkov3, A.V. Nikonorov" Liver Tumor Segmentation CT Data Based on Alexnet-Like Convolutional Neural Nets," Information Technology and Nanotechnology (ITNT-2016), vol. (2016), pp.348-356.

[11] V. Badrinarayanan, A. Kendall, R. Cupola "SegNet: A Deep Convolutional Encoder-Decoder Architecture for Image Segmentation "arXiv:00561v3. [cs.CV],10 oct 2016.

[12] M. Bellver, K.-K. Maninis, X. Giró-I-Nieto, J. Torres, L. Van Gool," Detection-Aided Liver Lesion Segmentation Using Deep Learning" arXiv: 1702.05747, 2017

[13] K. Simonyan and A. Zisserman, "Very deep convolutional networks for large-scale image recognition," arXiv preprint arXiv:1409.1556, 2014.

[14] E. Vorontsov, A. Tang, C. Pal, et al., "Liver lesion segmentation informed by joint liver segmentation", in: Proceeding of the 15th IEEE International Symposium on Biomedical Imaging, IEEE, 2018. pp. 98-136.

[15] O. Ronneberger, P. Fischer, and T. Brox,2015, "U-net: Convolutional networks for biomedical image segmentation", in: Proceeding of International Conference on Medical Image Computing and Computer. pp. 234-241.

[16] P. Bilic, P. F. Christ, E. Vorontsov, et al., "The liver tumor segmentation benchmark (LITS)," arXiv preprint arXiv:1901.04056, 2019.

[17] The LITS website (2017). [Online]. Available: https://competitions.codalab.org/competitions/17094.

[18] J. Long, E. Shelhamer, and T. Darrell, "Fully convolutional networks for semantic segmentation," in CVPR, pp. 3431-3440, 2015

[19] C. Nwankpa, W. Ijomah, A. Gachagan, A., S. Marshall "Activation functions: Comparison of trends in practice and research for deep learning". arXiv preprint arXiv:1811.03378,2018.

[20] R., Galina. "Combining the results of several neural network classifiers." Neural networks 7.5, pp. 777-781, 1994

[21] K. H. Zou, S. K. Warfield, A. Bharatha, C. M. Tempany, M. R. Kaus, S. J. Haker, and R. Kikinis, "Statistical validation of image segmentation quality based on a spatial overlap index1: scientific reports," Academic Radiology, vol. 11(2), pp. 178-189, 2004.

[23] N. A. Elmenabawy, A. Elnakib, H. Moustafa, 2020" Deep Joint Segmentation of Liver and Cancerous Nodules from CT Images", in: Proceeding of 37th National Radio Science Conference (NRSC 2020). [in press]
Title Arabic:

$$
\text { نظام آلي عميق لتجزئة الكبد والورم باستخدام تصويت الأغلبية }
$$

Arabic Abstract:

في هذ البحث، نقترح نظام قائم على التعلم المتعمق وتصويت الأغلبية للتجزئة

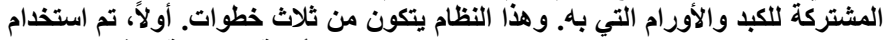

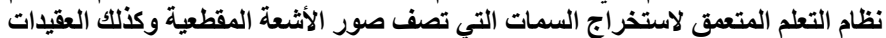

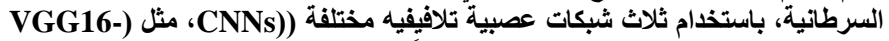
(Segnet, ED-Alexnet, Resnet-18

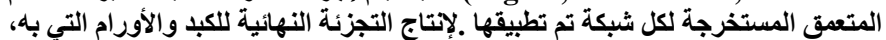

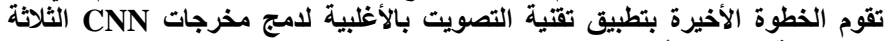

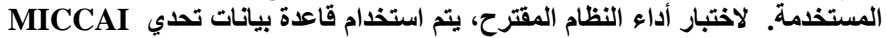

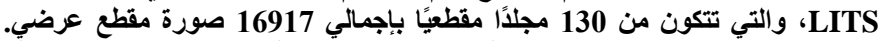

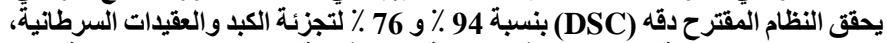

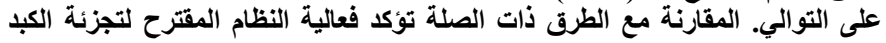

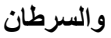

Nermeen Elmenabawy is a graduate student in Electronics and Communications Engineering (ECE) Department, Faulty of Engineering, Mansoura University since 2017. She received her BSc from the ECE Department in 2013. Her research interests include deep learning and medical image analysis. She has gained a two-year hand-on experience on medical data analysis during her research studies.

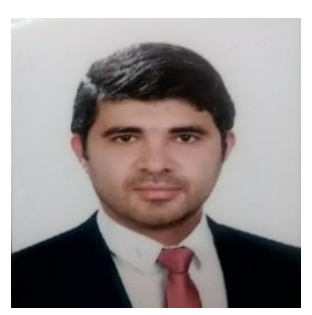

Ahmed Elnakib is an associate professor in ECE department, Mansoura Univeristy. D. Elnakib have authored or co-authored more than 25 journal articles, 7 book chapters, and 30 peer-reviewed conference papers. D. Elnakib is a regular reviewer for top international medical signal analysis journals that include: Medical Image Analysis, IEEE Transactions on Medical Imaging, and Neurocomputing. In 2013, he has awarded the John Houchens Prize for the best outstanding dissertation. In 2020, he won the state encouragement award in Engineering Sciences.

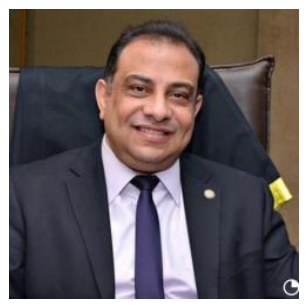

Hossam El-Din Moustafa is an associate Professor at the Department of Electronics and Communications Engineering, and the founder and executive manager of Biomedical Engineering Program (BME) at the Faculty of Engineering, Mansoura University. The main research points include biomedical image and signal processing and deep learning applications. 